\title{
MAGNETIC FIELDS IN THE DISK-HALO INTERFACE
}

\author{
Yoshiaki SOFUE \\ Institute of Astronomy, The University of Tokyo \\ Mitaka, Tokyo 181, Japan
}

\begin{abstract}
A review is given of large-scale magnetic fields in disks and halos of spiral galaxies. A particular attention is given to vertical field structures, and we discuss their origin and implication on their interaction with halo gas. We point out that the disk-halo magnetic interface plays an important role in circulation of interstellar gas in galaxies, in particular a large-scale circulartion from the galactic center to outer disk regions.
\end{abstract}

\section{INTRODUCTION}

Large-scale magnetic fields in spiral galaxies have been measured by observing their synchrotron polarized radio emission. Magnetic field energy density in spiral galaxies is usually comparable to thermal and kinetic energies of interstellar gases, which indicates that magnetic field plays a siginificant role in the behaviour of interstellar matter. Existence of the large-scale fields suggests that the field may affect a galaxy-scale circulation of gas. In some galaxies magnetic fields in halos are as strong as those in disks, suggesting that their halo gases are driven by a magnetic mechanism. In some galaxy nuclei, magnetic energy density exceeds that of gaseous kinetic energy density, which suggests that the gas dynamics is controled by magnetic fields.

In this paper we review magnetic fields in disks and halos of spiral galaxies. We pay particular attention to vertical magnetic fields in spiral galaxies and their central regions. We discuss a possible evolution of the primordially trapped galactic field, particularly the vertical component. We also discuss implication of vertical fields in an evolution of primeval galaxies. We show that the frozen-in and amplified vertical field in turn plays a role to accelerate the accretion of gas toward the center through the magnetic angular momentum transfer. We suggest that the vertical field near the galactic centers of primordial galaxies may be related to formation of a dense nuclear disk with starburst.

\section{DISK FIELDS IN SPIRAL GALAXIES}

Field Strength: Strength of magnetic field can be approximately determined from synchrotron radio intensity by assuming an equipartition between magnetic and cosmic-ray energy densities. Using thus obtained field strengths $B$ for galaxies, 
an empirical relation beteen magnetic energy density and molecular gas density $\rho$ in disk galaxies has been derived as follows:

$$
B^{2} / 8 \pi \propto \rho
$$

This relation can be rewritten to give field strength as a function of gas density as

$$
B / B_{0} \simeq \sqrt{\rho / \rho_{0}}
$$

with $B_{0} \simeq 3-5 \mu \mathrm{G}$ and $\rho_{0} \simeq 1 m_{\mathrm{H}} \mathrm{cm}^{-3}$.

Field Orientation and the Origin: Orientation of disk magnetic fields as measured from linear polarization observations and Faraday rotation analyses is either BSS (bisymmetric spiral) or a ring (see, e.g. Sofue et al 1986). In most cases, higher-order and/or random fields of comparable strengths are superposed.

The BSS fields may have their origin in a primordial magnetic field when galaxy formed: A larger-scale intergalactic/intrucluster field was trapped and wound up by differential rotation. The BSS field can be then maintained in a steady state by a dynamo mechanism (e.g., Sawa and Fujimoto 1986). If the primordial field had an anisotropy, even a ring field can be created by a reconnection in the inner disk (Sofue et al 1986).

It is difficult to creat a large-scale ring field only from small-scale local fields (such as due to supernovae) by dynamo amplification, because directions of dynamoamplified ring fields should reverse frequently from a radius to another. Therefore we need a seed field which had originally large-scale ring configuration. Combination of the primordial-origin ring field, which was created from an anisotropic component of the primordial field, and a dynamo (amplification and maintaining) may explain the observed large-scale ring configuration.

\section{HALO FIELDS IN SPIRAL GALAXIES}

Our Galaxy: Magnetic fields in our Galactic halo can be derived from Faraday rotation analysis of external radio sources and pulsars. If we plot $|R M|$ (rotation measure) against $|\cot b|$ for radio galaxies and quasars with $b$ being galactic latitude, the upper envelope of the plot can be fitted by a relation,

$$
|R M|_{\mathrm{RG}, \mathrm{Quasar}} \simeq 30|\cot b| \text {. }
$$

On the other hand if we plot the same for pulsars, we obtain

$$
|R M|_{\text {Pulsar }} \simeq 10|\cot b| \text {. }
$$

The difference between the coefficients for the two plots may be due to Faraday rotation in a space above a disk in which pulsars are distributed, namely it may be due to a halo beyond $0.5-1 \mathrm{kpc}$ from the galactic plane:

$$
|R M|_{\text {Halo }} \sim 20|\cot b| \text {. }
$$


If we take an electron density and a thickness of the halo to be approximately $10^{-3} \mathrm{~cm}^{-3}$ and a few kpc, respectively, the field strength in the halo is estimated to be a few $\mu \mathrm{G}$.

External galaxies: Observational data for halo fields in external galaxies are still crude. A few edge-on galaxies like NGC 4631 show extended nonthermal radio halo (e.g. Hummel et al 1988). NGC 4631 has a well ordered vertical halo field. The field strength from the radio emissivity is estimated to be a few $\mu \mathrm{G}$. There are many galaxies for which no evidence for radio halo is seen.

\section{VERTICAL FIELDS IN SPIRAL GALAXIES}

Vertical Radio Spurs - Magnetic Disk-Halo Interface: The radio continuum maps of the Milky Way show vertical structures emerging from the galactic plane (Sofue 1988). Their appearence parallel to each other suggests their coherent origin, likely driven by magnetic lines of force emerging normal to the disk plane. The vertical spurs are concentrated toward the inner region of the solar circle, suggesting that they are not local objects. Moreover, spurs are more often found above spiral arms than in interarm regions. This fact suggests that the spurs are manifestation of ejection of matter, including cosmic rays, from intense star forming regions in the spiral arms (Sofue and Tosa 1974; Sofue 1976). Their vertical extents are a few hundred pc to a kpc, while their widths are of the order of 100 pc.

Vertical Dust Lanes in External Galaxies: External edge-on galaxies often show vertical structures, which are most clearly seen as dust lanes. Long, coherent and thin filaments running normal to the disk of some dust-rich spirals like NGC 253 suggest the exitence of a large-scale vertical field running across the disk plane (Sofue 1987; Sofue et al 1990). The vertical structures are found not only in the central regions but also at radii of a few to $10 \mathrm{kpc}$, and run for more than a kiloparsec toward the halo, in some cases more than $3 \mathrm{kpc}$.

Vertical Magnetic Fields in the Galactic Center: In the inner region of our Galaxy direct evidence for a vertical field has been found by high-resolution radio observations of the synchrotron radio emission as well as by polarization observations. A large number of straight filaments extending for a hundred pc scale run almost perpendicular to the disk plane near the Arc and are well understood as the trace of a magnetic field running vertical to the disk (Yusef-Zadeh et al 1984). The radio Arc shows strong linear polarization, and Farday-roatation corected data directly indicates a poloidal magnetic field (Tsuboi et al 1986; Sofue et al 1987).

Vertical Magnetic Fields in Nuclei of External Galaxies: Polarization observations of the nuclear radio source in M31 shows the magnetic efield orientation perpendicular to the major axis (Berkhuijsen et al 1987). Since the galaxy is highly inclined, this may be attributed to a poloidal magnetic field in the nucleus. Besides M31, however, no obvious magnetic structures are known for nuclei in external galaxies mainly because of a lack of observations. 


\section{EVOLUTION OF MAGNETIC FIELDS IN GALAXIES}

Primordial Origin of Galactic Magnetic Fields: The BSS field configuration can be understood, if it is a fossil of an intergalactic and/or intracluster field wound up by the primordial galaxy disk. The disk field is then maintained in a steady state by the induction-dynamo mechanism (Fujimoto and Sawa 1987; Sawa and Fujimoto 1986). Even a ring field can be produced from the primordial one, if we allow for an initial asymmetry with respect to the center.

A vertical magnetic field trapped into a premeval galaxy is accreted toward the central region of the galaxy, producing there a strong vertical field (Sofue and Fujimoto 1987). It has been shown that a vertical field of strength of the order of a $\mathrm{mG}$ can be produced as the result of accretion of mass toward the center according to the viscous-inflow and on-going star formation model of primeval galaxies (Yoshii and Sommer-Larsen 1989; Yoshii and Saio 1990).

Vertical Primordial Magnetic Field: It is natural that a large-scale field component parallel to the rotation axis existed in addition to the disk field, when a galaxy formed. This field component is also trapped to the prime,val gas sphere. Since the disk radius is large enough and the diffusion time is longer than the galaxy evolution time, the vertical field is almost frozen into the disk gas. The vertical field then follows an evolution as described below (Sofue and Fujimoto 1987). Strength of a vertical field frozen into a protogalactic gas sphere varies with the radius as $B=B_{0}\left[\Sigma(r) / \Sigma_{0}\right]$, where $B_{0}$ is the primordial intergalactic magnetic field strength of the order of $B_{0} \sim 10^{-9} \mathrm{G}, \Sigma$ is the surface mass density of the galaxy, and $\Sigma_{0}$ its initial value before protogalactic contraction as givey by $\Sigma_{0} \sim M_{\mathrm{G}} /\left(\pi R_{0}^{2}\right)$. Here $M_{\mathrm{G}}$ is the total mass of the galaxy, and is given by $M_{\mathrm{G}} \sim(4 \pi / 3) R_{0}^{3} \rho_{0}$ with $\rho_{0}$ being the intergalactic gas density and $R_{0}$ the initial radius of the pre-contraction gas sphere. Then we obtain $\Sigma_{0} \sim(16 / 9 \pi)^{1 / 3} \rho_{0}^{2 / 3} M_{\mathrm{G}}^{1 / 3}$.

The surface density at radius $r$ in the formed galaxy can be written as $\Sigma \sim$ $V(r)^{2} /(\pi G r)$, where $V(r)$ is the rotation velocity at $r$ and $G$ is the gravitational constant. We thus obtain a field strength $B$ (in G) at the stage when the initial contraction of the gas sphere finished:

$$
B \sim 4.1 \times 10^{-5}\left(\frac{B_{0}}{10^{-9} \mathrm{G}}\right)\left(\frac{\rho_{0}}{10^{-5} \mathrm{~cm}^{-3}}\right)^{-2 / 3}\left(\frac{M_{\mathrm{G}}}{10^{11} M_{\odot}}\right)^{-1 / 3}\left(\frac{V}{200 \mathrm{~km} \mathrm{~s}^{-1}}\right)^{2}\left(\frac{r}{1 \mathrm{kpc}}\right)^{-1} .
$$

We here confirm that the magnetic field is passive against the gravitational contraction in this stage of primeval galaxy formation: In the stage of primeval galaxy contraction, when nearly all material was gas, the gas density is given approximately by $M(r) \sim(4 \pi / 3) \rho r^{3}$, and $M(r) \sim r V^{2} / G$. Thus a possible maximum field (in $\mathrm{G}$ ), below which the field is passive against gas motion, can be evaluated as

$$
B_{\max } \sim \sqrt{3 / G} V^{2} / r \sim 8.5 \times 10^{-4}\left(\frac{V}{200 \mathrm{~km} \mathrm{~s}^{-1}}\right)^{2}\left(\frac{r}{1 \mathrm{kpc}}\right)^{-1} .
$$

This value is much larger than the value estimated from the frozen-in formula, and we may naturally assume that the magnetic field in the protogalactic sphere (disk) 
is passive against the gas in gravitational contraction. We also note that the time scale for diffusion of the fertical field from the protogalaxy is more than $10^{12}$ years (see section 6), and the assumption of frozen-in field is a good one.

Exponential Disk in Primeval Galaxies and Stronger Field near the Center: If we take into account the formation of an exponential disk according to the viscosity-driven angular momentum transfer and on-going star formation model (Yoshii and Sommer-Larsen 1989), the central part of a galaxy gets stronger magnetic field. The magnetic flux conservation results in a radial distribution of the field strength obeying the exponential law. In the central few hundred pc region the gas density attains an excess by an order of magnitude over the value given by a simple exponential disk, and the field strength becomes correspondingly stronger, or of the order of $0.1-1 \mathrm{mG}$. The initial star formation then finishes when the gas is fed into stars and the density decreases to a certain threshold value, after which the magnetic field is no more frozen into the stellar disk.

Strong Vertical Fields near the Galactic Nuclei: The vertical magnetic field is then frozen into the gas left behind the initial star formation. At this stage the gas may have a constant threshold density below which the initial star formation did not take place, and shares about ten percent of the total mass. The "interstellar" gas then follows its own evolution goveraned by the density wave shock, cloud-cloud collisions, star formation, and/or magnetic accretion as described in the next section. In particular, in the central region, a bar-induced shock and the magnetic accretion will enhance the accretion. Since the diffusion time of the vertical field is shorter than the dynamical time scale, this results in a formation of a stronger vertical field in the center. If the present intergalactic or intracluster magnetic field is of the order of $10^{-9 \sim-10} \mathrm{G}$, the vertical field strength in the central $1 \mathrm{kpc}$ of normal spiral galaxies is expected to be of the order of a $\mathrm{mG}$.

\section{MAGNETIC ACCRETION AND GALACTIC MAGNETIC FOUN- TAIN}

Magnetic Accretion: The interstellar gas in turn suffers from a magnetic torque by the vertical field which is twisted by the galactic rotation. The time scale with which the rotating gas element loses angular momentum is given by $\tau=V r /\left(B^{2} / 4 \pi \rho_{\text {gas }}\right)$, where $\rho_{\text {gas }}$ is the interstelar gas density. This time scale is then calculated to be $\tau \sim 10^{11}, 10^{8}$, and $10^{5}$ years, respectively at $r=5,1$ and $0.1 \mathrm{kpc}$. This shows that the magnetic torque is not negligible in the inner region of thFe galaxy. The angular momentum of the disk gas is transfered and lost by the magnetic torque. Then the accretion of gas is accelerated, and as well the accretion of the vertical field is accelerated. This magnetic accretion proceeds until the magnetic energy density, $E_{\mathrm{m}}=B^{2} / 8 \pi$, becomes comparable to the gravitational/rotational energy density of gas, $E_{\mathrm{g}} \sim \rho_{\text {gas }} V^{2} / 2$.

After separation of interstellar gas from stars, the magnetic field is not necessarily passive against the gas motion. In the inner $1 \mathrm{kpc}$, for example, where the galactic-shock and/or bar-shock cause further accretion coupled with the magnetic accretion, the accretion proceeds until the magnetic energy becomes com- 
parable to the gravitational energy, or the accretion stops when $E_{\mathrm{m}} \sim E_{\mathrm{g}}$. For $\rho_{\text {gas }}=100 m_{\mathrm{H}^{\mathrm{cm}}}$ and $V \sim 200 \mathrm{~km} \mathrm{~s}^{-1}$ the magnetic accretion proceeds until the field strength becomes as strong as $\sim 1 \mathrm{mG}$.

The rapid accretion of gas toward the nucleus makes a dense nuclear gas disk, and will be related to starburst activity near the center. We emphasize that the magnetic accretion is effective to create a dense gas disk near the nucleus even without a bar potential and disturbance from companion galaxies, which is often suggeted for some interacting galaxies with starburst activity. We note that some non-interacting galaxies like NGC 253 show starburst activity.

Galactic Magnetic Fountain - Large-Scale Circulation: The twisted vertical magnetic field near the nucleus then accelerates a screwing outflow of gas, and results in a vertical jet from the nucleus (Shibata and Uchida 1988). This mechanism will explain many of the observed vertical radio features near the nuclei of spiral galaxies (Hummel et al 1983). The outflow transfers gases from the nuclear region into the halo. The gas in the disk is highly poluted by heavy elements as a result of atarburst activity, etc, so that this large-scale circulation may cause an overall polution of gas in a galaxy: by this large-scale outflow, which we call a "galactic magnetic fountain", heavy elements involved in the nuclear disk gas are spread over the entire galaxy via the halo magnetic fields.

\section{References}

Berkhuijsen, E.M., Beck, R., and Gräve, R. 1987, in Interstellar Magnetic Fields, ed. R. Beck and R. Gräve (Springer Verlag, Berlin), p.38.

Fujimoto, M., and Sawa, T. 1987, Publ. Astron. Soc. Japan, 39, 375.

Hummel,E., Lesch,H., Wielebinski,R., Schilickerser,R. 1988, Astron. Astrophys. Letters, 197, L29.

Hummel,E., Kotanyi,G.G., van Gorkom,J.H. 1983, Astrophys. J. Letters, 267, L5.

Sawa, T., and Fujimoto, M. 1986, Publ. Astron. Soc. Japan, 38, 133.

Shibata, K., and Uchida, Y. 1987, Publ. Astron. Soc. Japan, 39, 559.

Sofue, Y. 1976, Astron. Astrophys., 48, 1.

Sofue, Y. 1987, Publ. Astron. Soc. Japan, 39, 547.

Sofue, Y. 1988, Publ. Astron. Soc. Japan, 40, 567.

Sofue, Y., and Fujimoto, M. 1987, Publ. Astron. Soc. Japan, 39, 843.

Sofue, Y., Fujimoto, M., and Wielebinski, R. 1986, Ann. Rev. Astron. Astrophys., 24, 459 .

Sofue, Y., Reich, W., Inoue, M., and Seiradakis, J.H. 1987, Publ. Astron. Soc. Japan, 39, 359.

Sofue, Y.and Tosa, M. 1974, Astron. Astrophys., 36, 237.

Sofue, Y., Wakamatsu, K., Mallin, D.F., 1990, in this issue.

Tsuboi, M., Inoue, M., Handa, T., Tabara, H., Kato, T., Sofue, Y., and Kaifu, N. 1986, Astron. J., 92, 818.

Yoshii, Y., and Sommer-Larsen, J. 1989, Monthly Notices Roy. Astron. Soc., 236, 779.

Yusef-Zadeh, F., Morris, M., and Chance, D. 1984, Nature, 310, 557 